\section{Development of a multiscale testing method for the reduction of soft biological tissues waste during mechanical characterization}

\author{
Andrea T. Lugas,1,2 Gianpaolo Serino,1,2 \\ Mara Terzini, ${ }^{1,2}$ Cristina Bignardi, ${ }^{1,2}$ \\ Alberto L. Audenino ${ }^{1,2}$ \\ 1Department of Mechanical and \\ Aerospace Engineering, Politecnico di \\ Torino, Turin; 2 PolitoBIOMed Lab, \\ Politecnico di Torino, Turin, Italy
}

\begin{abstract}
Two biaxial mechanical test methods were devised to compare their suitability for the mechanical characterization of soft biological tissues with the least possible tissue waste. Nanoindentation was used to explore the microscopic properties of the tissue and to overcome the macroscopic test limitations.
\end{abstract}

\section{Introduction}

The bovine pericardium is a widely used biomaterial for the reconstruction of heart valves. ${ }^{1}$ Investigating the mechanical properties of the tissue is a crucial task for prostheses design, and it is usually performed through biaxial tensile tests. However, specimen variability is highly considered in specimen selection for tests, which causes the exclusion of most of the tissue.2,3 Furthermore, since the pericardium is stiffer in the collagen fibers direction $^{4,5}$ the Extracellular Matrix (ECM) structure is an important property to consider before macroscopic mechanical tests, and the nanoindentation of the surface can provide information about the fiber orientation.

This study aims to develop a standardized combined multiscale method to investigate biological soft tissue mechanical properties, limiting specimen-to-specimen variability and avoiding tissue waste.

\section{Materials and Methods}

Samples of bovine pericardium were decellularized and cut in $15 \times 15 \mathrm{~mm}^{2}$ square-shaped specimens. The thickness was measured through a digital caliper at 5 points in the central region of each square, and the mean value was considered as the thickness of the specimen. Nanoindentation tests were conducted in the central region of each specimen using a commercial nanoindenter (Piuma, Optics 11, Netherlands). Indentations were performed in a $50 \times 50$ matrix with a $200 \mu \mathrm{m}$ step size in each direction, resulting in a $10 \times 10 \mathrm{~mm}^{2}$ surface of indentation. The tests were performed using a probe with $4.36 \mathrm{~N} / \mathrm{m}$ stiffness and $43.5 \mu \mathrm{m}$ tip radius, and the indentation depth was set to $2000 \mu \mathrm{m}$.

A load-controlled and a displacementcontrolled protocol were devised and compared to investigate the more reliable macroscopic testing method. In both cases, sixteen equally spaced sutures were placed on the edges of the specimen and connected to four grip fixtures on the Planar Biaxial TestBench Instrument (TA Instruments, USA). Four small markers were drawn in the central region of each specimen before the test for optical strain computation (Figure 1), which is highly recommended to avoid mechanical interference. ${ }^{6}$ All nanoindentation and biaxial tensile tests were performed in wet conditions, soaking the specimens in a PBS bath at a temperature of $37^{\circ} \mathrm{C}$.

\section{Results}

The adopted technique allowed a rapid measurement of the thickness, which avoided excessive dehydration of the specimens during the process. The nanoindentation protocol provided a non-destructive method to compute the surface elastic modulus of the tissue.

The load-controlled test resulted not repeatable and hard to control because of the high specimen variability. Such type of control, indeed, requires specific Proportional-Integrative-Derivative (PID)
Correspondence: Andrea T. Lugas, Department of Mechanical and Aerospace Engineering, Politecnico di Torino, Turin; PolitoBIOMed Lab, Politecnico di Torino, Turin, Italy.

E-mail: andrea.lugas@polito.it

Key words: Soft biological tissues; biaxial mechanical tests; nanoindentation; multiscale characterization.

Disclosures: The authors declare no conflict of interests.

Conference presentation: This paper was presented at the Third Centro 3R Annual Meeting - L'era delle 3R: modelli in silico, in vitro e in vivo per promuovere la ricerca traslazionale 30 September - 1 October 2021, Evento online organizzato dal Politecnico di Torino.

Received for publication: 9 July 2021.

Accepted for publication: 7 September 2021.

This work is licensed under a Creative Commons Attribution NonCommercial 4.0 License (CC BY-NC 4.0).

(C) Copyright: the Author(s), 2021

Licensee PAGEPress, Italy

Biomedical Science and Engineering 2021; 4(s1):176 doi:10.4081/bse.2021.176

control parameters, which are computed through a tuning on the specimen before the test. However, the parameters computed with the tuning, could not be valid for other specimens, depending on their characteristics (e.g. the thickness and the fiber orientation). This caused instability of the control system and the consequent unexpected destruction of some specimens. Since a specimen-specific tuning was not necessary

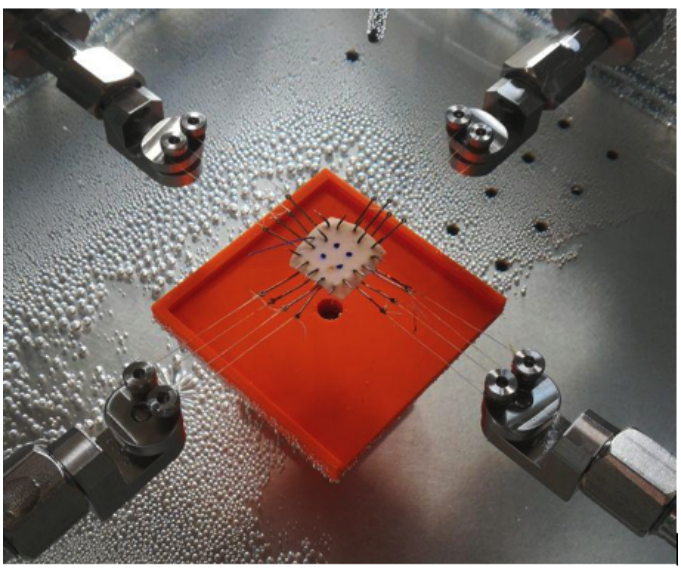

Figure 1. Pericardium specimen test setup. 
to obtain a stable control, the displacementcontrolled test resulted to be more repeatable and easier to exploit. The test consisted of five equibiaxial (i.e. with the same displacement in the two planar directions) preconditioning cycles of elongation, followed by five cycles with different displacements applied along the two directions (Figure 2).

\section{Discussion and Conclusions}

This work provided a reliable mechani- cal multiscale testing protocol for bovine pericardium and soft biological tissues, which can be exploited for future investigations and constitutive modeling with a diminished waste of animal biological material. Further improvement of the protocol may be obtained considering an optic technique for determining material axes (i.e. the collagen fiber orientation), such as Small-Angle Light Scattering (SALS). Furthermore, more accurate optical strain computation could be achieved using the Digital Image Correlation system (DIC).

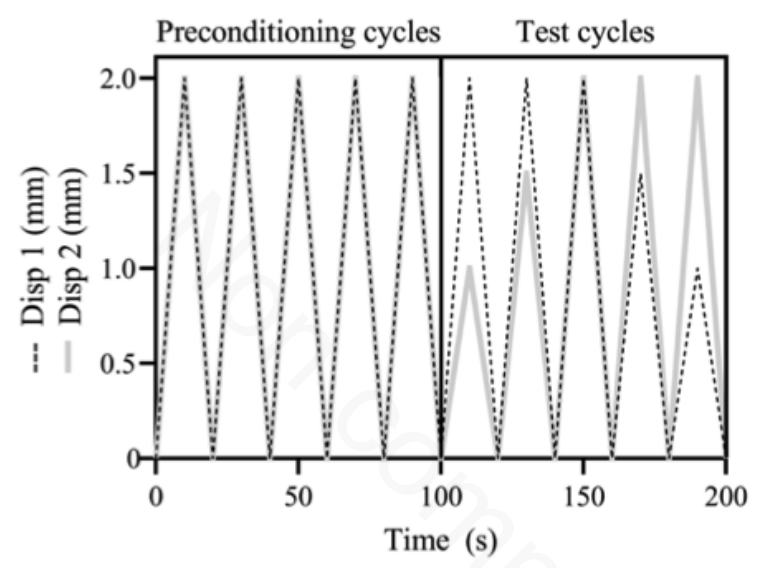

Figure 2. Biaxial testing protocol. Disp 1 and Disp 2 are the displacements applied along the preferred and cross-preferred fiber orientations, respectively.

\section{References}

1. Negishi J, Hashimoto Y, Yamashita A, Zhang Y, Kimura T, Kishida A, Funamoto S. Evaluation of small-diameter vascular grafts reconstructed from decellularized aorta sheets. J Biomed Mater Res A 2017;105:1293-8.

2. Hiester ED, Sacks MS. Optimal bovine pericardial tissue selection sites. I. Fiber architecture and tissue thickness measurements. J Biomed Mater Res 1998;39:207-14.

3. Hiester ED, Sacks MS. Optimal bovine pericardial tissue selection sites. II. Cartographic analysis. J Biomed Mater Res 1998;39:215-221.

4. Billiar KL, Sacks MS. Biaxial mechanical properties of the natural and glutaraldehyde treated aortic valve cuspPart I: Experimental results. J Biomech Eng 2000;122:23-30.

5. Sun W, Sacks MS, Sellaro TL, et al. Biaxial mechanical response of bioprosthetic heart valve biomaterials to high in-plane shear. ASME. J Biomech Eng 2003;125:372-80.

6. Sacks MS, Sun W. Multiaxial mechanical behavior of biological materials. Annu Rev Biomed Eng 2003;5:251-84. 